Les interactions entre élèves médiatisées par des indices comme facteur déclencheur de progrès collectifs et individuels en situation d'apprentissage coopératif face à une tâche complexe de mathématiques

Interactions between students mediated by cues as activating factors of collective and individual progress in cooperative learning situations when dealing with a complex mathematical task Las interacciones entre los alumnos mediatizadas con indicaciones como factor activador de progresos colectivos e individuales en situación de aprendizaje cooperativo en una actividad compleja en matemáticas

Annick Fagnant, Isabelle Demonty, Émilie Lemaire et Matthieu Scheen

Volume 47, numéro 3, automne 2019

Les interactions sociales au service des apprentissages mathématiques

URI : https://id.erudit.org/iderudit/1066512ar

DOI : https://doi.org/10.7202/1066512ar

Aller au sommaire du numéro

Éditeur(s)

Association canadienne d'éducation de langue française

ISSN

1916-8659 (numérique)

Découvrir la revue

\section{Citer cet article}

Fagnant, A., Demonty, I., Lemaire, É. \& Scheen, M. (2019). Les interactions entre élèves médiatisées par des indices comme facteur déclencheur de progrès collectifs et individuels en situation d'apprentissage coopératif face à une tâche complexe de mathématiques. Éducation et francophonie, 47(3), 35-57.

https://doi.org/10.7202/1066512ar
Résumé de l'article

Cette étude exploratoire s'appuie sur une analyse qualitative des interactions entre élèves confrontés à la résolution d'une tâche complexe de mathématiques lors d'un travail de groupe structuré selon les principes de l'apprentissage coopératif. Plus précisément, elle cherche à voir l'impact des régulations interactives entre élèves, médiatisées par des indices visant à soutenir les processus de représentation et de vérification, sur les performances collectives (la résolution de la tâche complexe durant le travail de groupe) et individuelles (la résolution d'une tâche de réinvestissement, à réaliser seul après le travail de groupe). L'enjeu de l'article est non seulement de donner à voir la potentielle richesse des interactions entre élèves, mais aussi de pointer les difficultés liées au travail de groupe, et ceci même quand celui-ci a été conçu en s'appuyant sur une approche méthodologique (l'apprentissage coopératif) dont l'efficacité a été largement démontrée.
Tous droits réservés (C) Association canadienne d'éducation de langue française, 2019 cest protége par la loi sur le droit d'auteur. L'utilisation des services d'Érudit (y compris la reproduction) est assujettie à sa politique d'utilisation que vous pouvez consulter en ligne. 


\title{
Les interactions entre élèves médiatisées par des indices comme facteur déclencheur de progrès collectifs et individuels en situation d'apprentissage coopératif face à une tâche complexe de mathématiques
}

\author{
Annick FAGNANT \\ Université de Liège, Belgique \\ Isabelle DEMONTY \\ Université de Liège, Belgique \\ Émilie LEMAIRE \\ École libre fondamentale Saint-Joseph de Herve, Belgique \\ Matthieu SCHEEN \\ Université de Liège, Belgique
}

\section{RÉSUMÉ}

Cette étude exploratoire s'appuie sur une analyse qualitative des interactions entre élèves confrontés à la résolution d'une tâche complexe de mathématiques lors d'un 
travail de groupe structuré selon les principes de l'apprentissage coopératif. Plus précisément, elle cherche à voir l'impact des régulations interactives entre élèves, médiatisées par des indices visant à soutenir les processus de représentation et de vérification, sur les performances collectives (la résolution de la tâche complexe durant le travail de groupe) et individuelles (la résolution d'une tâche de réinvestissement, à réaliser seul après le travail de groupe). L'enjeu de l'article est non seulement de donner à voir la potentielle richesse des interactions entre élèves, mais aussi de pointer les difficultés liées au travail de groupe, et ceci même quand celui-ci a été conçu en s'appuyant sur une approche méthodologique (l'apprentissage coopératif) dont l'efficacité a été largement démontrée.

\section{ABSTRACT}

Interactions between students mediated by cues as activating factors of collective and individual progress in cooperative learning situations when dealing with a complex mathematical task

Annick FAGNANT, University of Liège, Belgium

Isabelle DEMONTY, University of Liège, Belgium

Émilie LEMAIRE, Saint Joseph Free Basic School of Herve, Belgium

Matthieu SCHEEN, University of Liège, Belgium

This exploratory study is based on a qualitative analysis of interactions between students asked to solve a complex mathematical problem in groups structured according to the principles of cooperative learning. Specifically, it seeks to understand the impact of interactive regulation between students, mediated by cues to support processes of representation and verification, on collective performance (solving the complex problem during group work) and individual performance (solving a reinvestment task, to be done alone after group work). The aim of the article is not only to show the potential richness of interactions between students, but also to point out the challenges inherent in group work, even when it has been designed according to a methodological approach (cooperative learning) whose effectiveness has been widely demonstrated. 


\section{RESUMEN}

\section{Las interacciones entre los alumnos mediatizadas con indicaciones como factor activador de progresos colectivos e individuales en situación de aprendizaje cooperativo en una actividad compleja en matemáticas}

Annick FAGNANT, Universidad de Lieja, Bélgica

Isabelle DEMONTY, Universidad de Lieja, Bélgica

Émilie LEMAIRE, Escuela libre fundamental Saint-Joseph de Herve, Bélgica

Matthieu SCHEEN, Universidad de Lieja, Bélgica

El presente estudio exploratorio se apoya en un análisis cualitativo de las interacciones entre alumnos confrontados con la solución de una actividad compleja en matemáticas durante un trabajo de grupo estructurado según los principios del aprendizaje cooperativo. Más precisamente, se trata de ver el impacto de las regulaciones interactivas entre los alumnos, mediatizadas con indicaciones que buscan apoyar el proceso de representación y verificación, sobre los resultados colectivos (resolución de la actividad compleja durante el trabajo de grupo) e individuales (resolución de una actividad de re-inversión, que se realiza solamente después del trabajo de grupo). El reto de este artículo es no solamente mostrar la riqueza potencial de las interacciones entre los alumnos, sino también señalar las dificultades relacionados con el trabajo de grupo, y esto incluso cuando fue concebido apoyándose en un acercamiento metodológico (el aprendizaje cooperativo) cuya eficacia ha sido ampliamente demostrada.

\section{INTRODUCTION}

Dans l'enseignement primaire, il est largement reconnu que la résolution de problèmes implique un "processus complexe de modélisation mathématique» (Van Dooren, Verschaffel, Greer, De Bock et Crahay, 2015) dont la mise en œuvre pose d'importantes difficultés, tant aux élèves qui doivent le déployer qu'aux enseignants et enseignantes qui doivent les soutenir dans cette démarche (Fagnant, Marcoux et Vlassis, 2014). Du côté des élèves, les difficultés liées à la construction d'une représentation adéquate de la situation (Thevenot, Barouillet et Fayol, 2015) et au développement de stratégies métacognitives de vérification (Mevarech et Kramarski, 2014) ont largement retenu l'attention des chercheuses et des chercheurs. Du côté des enseignants et enseignantes, plusieurs travaux ont montré que ceux-ci se sentaient quelque peu démunis pour gérer ce type d'apprentissages en classe. Quelques-uns court-circuitent le raisonnement des élèves en commençant par une analyse collective des tâches (Ginsburg, Cooke, Leinwand et Pollock, 2005) ou en clôturant la 
leçon par des corrections collectives guidées par leur logique d'adulte (Demonty et Fagnant, 2014; Fagnant, Dupont et Demonty, 2016). D'autres, sans doute par peur de «résoudre à la place de l'élève», ne se sentent pas autorisés à mettre en œuvre un "étayage» soutenant la résolution de problèmes lors des phases individuelles ou de groupe (Mottier Lopez, 2012), ou réalisent un cadrage «trop étroit» permettant aux élèves de réussir sans comprendre (Bonnery, 2009).

Dans cette configuration, le développement de dispositifs innovants, permettant de soutenir les enseignants et enseignantes dans leurs approches didactiques visant à promouvoir le développement de compétences en résolution de problèmes auprès de leurs élèves, demeure une problématique d'actualité. Plusieurs travaux ont montré l'efficacité d'approches impliquant les enseignants et enseignantes dans des recherches quasi expérimentales les conduisant à développer un enseignement explicite de stratégies cognitives et métacognitives de résolution de problèmes (Hanin et Van Nieuwenhoven, 2016; Jaegers, Fagnant et Lafontaine, 2016), ou misant sur la création de dispositifs structurés de type tutorat (Crahay, Lebe et Hindryckx, 2001; Sandron et Fagnant, 2017) ou travaux de groupes (Buchs, Lerhaus et Crahay, 2012; Mevarech et Kramarski, 2014; Slavin et Lake, 2008). S'inscrivant dans cette seconde perspective, la présente étude exploratoire vise à analyser l'efficacité de travaux de groupes structurés selon les principes de l'apprentissage coopératif de façon à laisser la résolution de problèmes aux mains des élèves, tout en dynamisant les échanges entre ceux-ci grâce à des indices visant à soutenir les processus de représentation et de vérification. S'appuyant sur une analyse des interactions entre élèves, les objectifs de l'étude sont, d'une part, d'identifier les éléments qui permettent à des groupes d'élèves de résoudre ensemble une tâche complexe de mathématique qu'ils n'avaient pas pu résoudre seuls (progrès collectif) et, d'autre part, de repérer, parmi ces éléments, les déclencheurs d'un réel apprentissage permettant à chaque membre du groupe de réinvestir ses acquis dans une autre tâche du même type (progrès individuel).

\section{CADRE THÉORIQUE}

L'apprentissage coopératif a fait l'objet de nombreuses études, de méta-analyses et d'articles de synthèse (Hattie, 2009; Slavin et Lake, 2008). Son efficacité, tant sur les variables cognitives que motivationnelles, a été démontrée par un nombre important de recherches empiriques menées à différents niveaux scolaires et dans différentes disciplines (Buchs, Lerhaus et Crahay, 2012; Gillies, 2016; Plante, 2012).

Il ne suffit évidemment pas de mettre les élèves en groupes pour qu'ils tirent parti des interactions entre eux (Lerhaus, 2002; Gillies, 2016). L'apprentissage coopératif est une forme de travaux de groupes qui, misant notamment sur l'interdépendance entre les partenaires, prend une forme très structurée. Il se distingue d'autres formes de travaux de groupes, comme l'apprentissage collaboratif, notamment, qui, se présentant 
sous une forme moins structurée, mise davantage sur le partage et la collaboration volontaire, et laisse, en quelque sorte, l'investissement individuel à la discrétion de chacun (Baudrit, 2007). Alors que l'apprentissage collaboratif peut présenter un caractère plus spontané et permettre aux membres du groupe d'interagir avec plus de liberté, l'activité collective sera très soigneusement préparée dans une perspective d'apprentissage coopératif: les interactions entre élèves seront structurées pour «contraindre» le réel investissement de chacun. Quelques-uns évoquent l'idée de «scripts», explicitant la façon dont les interactions entre élèves doivent s'enchaîner et se soutenir tout au long de la tâche. Ces scripts semblent dès lors permettre des régulations interactives plus efficientes et le développement de régulations métacognitives (Chen et Chui, 2016).

Pour décrire les caractéristiques de l'apprentissage coopératif, Buchs et al. (2012) font référence aux cinq principes de base énoncés à la fin des années 1990 par Davidson: (1) la nécessité de proposer une tâche commune qui « exige la contribution de chaque membre du groupe pour l'élaboration du produit final [...] et que personne ne devrait être en mesure de résoudre sans l'apport des autres» (p. 442); (2) la constitution de groupes restreints, généralement composés de 3 à 5 personnes et constitués de façon à assurer une certaine hétérogénéité des partenaires; (3) le développement d'attitudes coopératives qui peuvent d'ailleurs faire l'objet d'un enseignement explicite préalable; (4) la nécessité de contraindre une «interdépendance positive»; et (5) une «responsabilisation individuelle». Ces deux dernières conditions constituent les éléments les plus saillants de l'apprentissage coopératif et on les retrouve explicitement pointées dans la plupart des écrits portant sur cette approche (Baudrit, 2007; Buchs et al., 2012; Gilies, 2016; Plante, 2012; Slavin, 2015). Il convient dès lors d'y accorder quelques lignes supplémentaires.

Tout d'abord, précisons que «l'interdépendance positive existe lorsque les élèves perçoivent qu'ils sont liés à leurs partenaires et qu'ils ne peuvent réussir à moins que leurs camarades réussissent (et réciproquement); les efforts de tous les équipiers doivent être coordonnés pour accomplir la tâche» (Buchs et al., 2012, p. 444). Cette «interdépendance» peut notamment être contrainte en agissant sur les "moyens» (Buchs et al., 2012), par exemple les «ressources» (ex.: se partager les informations à lire pour étudier une thématique); sur «les tâches» (ex.: corriger un texte commun en se focalisant sur des aspects différents) ou encore sur "les rôles" (ex.: prescrire ce que les membres de l'équipe sont en droit d'attendre de leurs coéquipiers et assigner des responsabilités à chacun). La «responsabilisation individuelle» comporte quant à elle deux aspects: "(1) chaque membre du groupe est responsable de son propre apprentissage; (2) chaque membre du groupe est responsable d'aider ses partenaires à apprendre» (Abrami, 1996, p. 87). Elle peut notamment être établie en s'assurant que les «résultats» de l'apprentissage individuel sont clairement reconnaissables et que les élèves s'en sentent responsables; que les «moyens» utilisés pour obtenir ces résultats exigent la participation de chacun ou encore que les interactions soient organisées de façon à responsabiliser chacun (Abrami, 1996). 
En plus des caractéristiques susmentionnées, plusieurs auteurs (Gillies, 2016; Plante, 2012) insistent sur un critère de "promotion des interactions" qui serait satisfait «lorsque les élèves issus d'une équipe de travail encouragent les efforts mutuels afin d'atteindre les buts fixés pour le groupe» (Plante, 2012, p. 256), et qui peut se manifester «lorsque les coéquipiers s'entraident, échangent des ressources [...] se fournissent des rétroactions constructives, questionnent le raisonnement et les conclusions des autres [...] et se font confiance» (Plante, 2012, p. 256). Les «scripts» précédemment évoqués (Chen et Chui, 2016) peuvent notamment aider à développer la «responsabilisation individuelle» et la "promotion d'interactions», en précisant les attentes vis-à-vis de chacun des membres du groupe, non seulement pour résoudre la tâche commune qui leur est proposée, mais aussi pour s'assurer que tous les membres du groupe comprennent bien les choix posés, les démarches développées et les solutions obtenues.

Lorsque les élèves travaillent en groupe, on fait l'hypothèse que les interactions interindividuelles seront sources de progrès intra-individuel en misant sur la régulation sociocognitive de conflits (Buchs, Darnon, Quiamzade, Mugny et Butera, 2008) ou, selon la loi de double-formation de Vygotsky, sur l'appropriation de processus cognitifs plus complexes, au départ accessibles uniquement dans le cadre d'interactions sociales, avant de devenir une conquête propre au sujet (Slavin, 2015). Plus largement, on peut aussi considérer qu'autrui constitue une source d'hétérorégulation (régulations interactives entre élèves) pouvant soutenir le processus autorégulateur de l'apprenant (Mottier Lopez, 2012). Dans l'étude qui nous occupe, les régulations interactives entre élèves sont également soutenues par des «outils» (régulations élèves-outils selon la typologie d'Allal, 2007), concrétisés par des indices centrés sur les processus de représentation et de vérification.

Quel que soit le modèle théorique auquel on fait référence pour chercher à comprendre comment les interactions entre élèves peuvent être source d'apprentissage (voir Slavin, 2010 pour une synthèse), encore faut-il décider quelles sont les «traces" qui permettront d'attester de ces progrès. Dans les études quasi expérimentales, les progrès observés dans des classes mettant en œuvre l'apprentissage coopératif sont comparés à ceux observés dans des classes développant des méthodes plus individuelles, voire compétitives (Hattie, 2009; Slavin et Lacke, 2008). D'autres travaux, plus qualitatifs, s'attachent à une analyse fine des hétérorégulations (entre l'enseignant ou l'enseignante et les élèves, ou entre élèves) et cherchent les «traces» d'un processus d'autorégulation auprès des élèves dans l'évolution de leur discours et des réponses qu'ils produisent (Demonty et al., 2014; Mercier Brunnel, 2017; Mottier Lopez, 2015). Dans l'étude présentée ici, en plus de l'analyse des interactions entre les élèves, médiatisées par les «indices» (c.-à-d. l'analyse de l'impact des hétérorégulations sur l'évolution du mode de raisonnement des élèves lors des travaux de groupes), nous chercherons à approcher la problématique de l'autorégulation en examinant les capacités démontrées par les élèves pour refaire seuls une tâche du même type que celle qu'ils ont résolue collectivement. 


\section{DISPOSITIF ET QUESTIONS DE RECHERCHE}

Les données présentées dans cet article ont été récoltées dans le cadre d'une étude plus large, réalisée par l'une des coauteures de cet article (Lemaire, 2016) dans le cadre de son travail de fin d'études en master en Science de l'Éducation à l'Université de Liège. Cette étude (non publiée) avait pour objectif de comparer l'efficacité respective de trois formes de travaux de groupe: groupes non structurés, groupes structurés en contraignant une interdépendance des rôles et groupes structurés via une interdépendance des tâches. L'étude a été réalisée dans quatre classes de $6^{\mathrm{e}}$ année primaire (grade 6 - élèves de 11-12 ans). Les élèves ont tout d'abord été confrontés à la résolution d'une tâche complexe qu'ils devaient résoudre de manière individuelle (voir annexe 1). Sur la base des résultats de ce test, trois groupes hétérogènes ont été constitués au sein de chacune des classes et répartis au hasard dans une des trois modalités précitées. Chaque groupe était composé de trois élèves ayant résolu entre zéro et deux sous-tâches (aucun n'avait pu résoudre l'entièreté de la tâche complexe). Au total, ce sont donc 12 groupes qui ont pu être observés à raison de 4 groupes par modalité. Chaque groupe recevait la même tâche que celle résolue individuellement et était informé qu'aucun élève du groupe n'avait pu la résoudre correctement dans son entièreté. Les groupes recevaient aussi des consignes pour travailler ensemble et des indices pour soutenir les processus de représentation et de vérification. La durée du travail de groupe n'était pas contrainte temporellement et variait entre 30 et 50 minutes. Tous les groupes ont été filmés et les échanges entre les membres ont été retranscrits. Une semaine après la phase de travail en groupe, les élèves ont reçu une tâche parallèle à celle résolue collectivement, et il leur a été demandé de la résoudre seul (tâche de réinvestissement).

Dans le cadre du présent article, nous avons fait le choix de nous centrer sur une analyse approfondie d'une des trois modalités de travaux de groupes, à savoir celle basée sur un apprentissage coopératif structuré par une interdépendance des rôles. La présentation des résultats des quatre groupes ainsi ciblés est guidée par deux questions de recherche: (1) comment les interactions entre élèves, médiatisées par les indices, permettent-elles au groupe de résoudre les nœuds mathématiques impliqués dans la tâche complexe? (2) les élèves qui ont pu résoudre correctement la tâche complexe en groupe parviennent-ils à réinvestir ces acquis collectifs dans un travail individuel du même type? En nous appuyant sur une analyse des transcriptions, nous cherchons à analyser l'impact des hétérorégulations sur l'évolution du mode de raisonnement des élèves lors des travaux de groupes, tout en recherchant les traces permettant de comprendre leur potentiel d'autorégulation se traduisant ici par la réussite de la tâche de réinvestissement en individuel. Pour comprendre les analyses qui vont suivre, il est important de se référer à l'annexe $\mathbf{1}$ qui présente la tâche complexe, les nœuds mathématiques à prendre en compte pour la résoudre et les indices de représentation et de vérification fournis aux élèves. Les consignes données au groupe concernant la répartition des rôles et les éléments explicitant la façon dont 
les élèves doivent travailler ensemble et se soutenir mutuellement (cf. idée de script) sont accessibles à l'annexe 2.

\section{RÉSULTATS}

Tout d'abord, notons que 3 groupes sur 4 ont pu résoudre correctement l'ensemble de la tâche complexe, le quatrième groupe ayant résolu correctement les deux premières sous-tâches, mais ayant échoué à la troisième. Au niveau de la tâche de réinvestissement, seul un groupe a atteint pleinement l'objectif en permettant à chacun des membres de résoudre seul une tâche comparable. L'analyse des échanges entre élèves au sein de chacun des groupes, orientée par les deux questions de recherche susmentionnées, va permettre de mieux comprendre ces résultats. Étant donné que les difficultés rencontrées par les élèves se situent au niveau des sous-tâches 2 et 3 , ce sont sur ces deux sous-tâches que nous allons centrer les analyses.

Dans le groupe le plus efficace (résolution correcte de la tâche lors du travail en groupe, puis par chacun des membres lors de la tâche de réinvestissement en individuel - G1), tous les élèves participent de manière assez équivalente, chacun étant relativement consciencieux dans son rôle. Au niveau de la sous-tâche 2, l'indice de représentation ne permet pas de résoudre la difficulté liée à l'intervalle et le groupe s'engage d'abord dans une démarche erronée (mesurer $10 \mathrm{~cm}$ entre les deux extrémités et conclure qu'il faut 10 plantes). C'est la prise en compte de l'indice de vérification qui permet de redynamiser les échanges et d'appréhender le nœud mathématique (voir transcription 1 - c'est sur la base de l'indice que Ga constate que la réponse « 10 » est erronée). On note que les élèves se souviennent des réponses qu'ils avaient produites lors de la résolution individuelle précédant le travail de groupe, mais qu'ils ne se fient pas totalement à celles-ci. Par ailleurs, il faut aussi souligner que les transcriptions montrent que l'élève qui a compris prend soin d'expliquer la démarche aux autres et de s'assurer de leur compréhension (Lé témoigne de son incompréhension, Di explique sa démarche, puis Ga reformule et Lé conclut qu'il a compris). Une fois le nombre de plantes trouvé, la résolution s'avère aisée puisqu'il n’y pas de réel nœud mathématique pour calculer le prix. L'indice de vérification est remobilisé pour s'assurer que la solution obtenue ne se trouve pas parmi les erreurs ciblées. 


\section{Transcription 1. Extraits des échanges du groupe 1 relatifs à la sous-tâche 2}

- Di: «0n a faux. 6,95 x 10.»

- Ga: "Ah oui, on a faux.»

$[\ldots]$

- Lé: "Pourquoi?»

- Ga: «Euh parce que ... Ah si, c'est parce que avant, on avait mis le zéro de la latte là et que zéro, on ne peut pas le compter. Enfin, je ne sais pas.»

- Di: "Moi, je ne me souviens plus comment j'avais fait mais j'avais compté 11 la dernière fois. »

- Ga: «Moi, j'avais mis 10 donc j'ai eu faux. »

- Lé: «Moi aussi, j'avais mis 10.»

- Ga : «Si tu avais mis 11, c'est que c'est juste, Di. »

- Lé: «Peut-être pas... Et pourquoi on ne partirait pas de là et qu'on compterait jusque-là?» [En montrant les extrémités sur l'indice de représentation.]

- Di : «Regardez [II place le 0 de sa latte sur le premier point représenté et commence à compter.]: 1 , $2,3 \ldots 10,11$. Ah ben, voilà pourquoi j'étais arrivé à 11. »

- Ga: "Bah, c'est 11 alors. Allais hop, on gomme. »

- Lé: «Mais oui, mais je ne comprends pas. Pourquoi tu as fait ça?»

- Ga: «Vas-y, fais un peu 6,95 x 11. » [En s'adressant à Lé.]

- Di : "Lé, attends, je vais t'expliquer. » [II prend le plan et la latte.] «Regarde je mets le 1 de la latte sur le point qui représente le $1^{\text {er }}$ forsythia, et puis après tu comptes jusqu'au $2^{\mathrm{e}}$ point. Donc ça fait $1,2, \ldots 10,11 . »$

- Ga: [Reformule l'explication de Di.] «Si on met le zéro de la latte, ça ne fait pas 1 mais zéro. C'est pour cela, qu'on met le 1 sur le point.»

- Lé: «Ah ok, j’ai compris.»

Lors de la sous-tâche 3, l'indice de représentation conduit à réaliser un quadrillage et les échanges entre élèves permettent de surmonter le premier nœud mathématique de cette sous-tâche (l'aire du triangle est calculée en reportant des carrés et des demi-carrés - voir transcription 2). Le nombre total de plantes est calculé en comptabilisant 20 plantes par carré et 10 plantes pour le dernier demi-carré. Un élève comprend rapidement qu'il convient d'acheter 6 paquets de 15 plantes, mais les deux autres peinent à suivre son raisonnement. Même s'il montre certains signes d'impatience, l'élève qui a trouvé la solution prend le temps d'expliquer sa démarche en repartant du point de départ (les deux premiers nœuds sont à nouveau explicités - les transcriptions montrent que Di explique à Ga, tout en faisant intervenir Lé, jusqu'à ce que Ga témoigne de sa compréhension); le seul élément qui n'est pas clairement réexpliqué concerne la façon dont il a trouvé qu'il fallait 6 paquets ( $3^{\mathrm{e}}$ nœud), mais les deux autres élèves ne manifestent plus de signe d'incompréhension. Au bout du compte, la consultation de l'indice de vérification permet de constater que la solution obtenue ne se trouve pas parmi les erreurs pointées, ce qui conforte les élèves dans leur démarche. 


\section{Transcription 2. Extraits des échanges du groupe 1 relatifs à la sous-tâche 3}

- Lé: «Regardez, mais attendez que je le fasse. Je vais dessiner le même carré qu'on a sur l'indice. Et puis un deuxième. Ce n'est pas très précis mais bon, on voit."

- Ga : «Oui ok.»

- Lé: "Donc maintenant, quand ils sont tous tracés, il reste 1, 2, 3.» [En montrant les 3 demi-carrés représentés dans le triangle.]

- Ga: "Ah oui, donc si on met ces deux-là ensemble, ça fait 1. »

- Lé: "Ça fait 4.»

- Di : "Et puis il reste celui-là » en montrant un demi-carré.

$[\ldots]$

- Di : «Ici, c'est déjà la moitié donc ce n'est pas 20 mais c'est 10. » [En montrant le demi-carré.]

- Lé: "Heu, je ne comprends plus ...»

- Di : «Regarde ici, c'est 20. Là, c'est 20, là c'est 20, avec ces deux-là, c'est 20. Et ici, comme c'est la moitié de 20, c'est 10.»

- Lé: "Bah oui, ça pourrait être logique. Donc 2, 4, 6, 8, 90 ».

- Ga: "Mais vous êtes sûrs que c'est la moitié d'un déjà?» [Et elle regarde avec sa latte.]

- Lé: «Oui, mais c'est juste que je n'étais pas très précise.»

- Di utilise la calculatrice et dit: «6x15 ça fait $90 »$.

[Les filles ne semblent pas prêter attention à ce qu'il dit.]

- Di: "Moi, j'ai trouvé quelque chose. 6 x 15 ça fait 90 et ça peut très bien nous aider. »

- Lé: «Quoi?».

- Di: " 6 paquets de 15, ça fait 90 donc pile poil ce qu'on a besoin. »

- Ga: «Bah non, t'as rien compris toi!»

- Di remontre sur le plan: «20,40,60, 80 si on prend les deux demis et avec celui-là 90 . Et 6 x 15, ça fait 90.»

- Lé lui prend la calculatrice et dit: «0n va voir $(6 \times 15)$. » Puis il dit: « Oh, il a tout juste, c'est bien! »

- Ga: «0n n'a pas besoin de 90. Pourquoi on aurait besoin de 90?»

[Lé et Di se tapent la tête d'une main comme pour dire, on vient de l'expliquer 3x.]

- Lé: "Pour une fois que j'ai compris un truc, on pourrait me féliciter.»

- Di : "Bon Ga, écoute moi et regarde. " [II retrace les carrés effacés à main levée dans le triangle.]

- Lé continue le tracé de Di et dit: «Y a 20 narcisses + 20 narcisses, ça fait $40+20$ narcisses, ça fait 60. Plus celui-là et celui-là, ça fait encore +20 et ... » [En montrant et écrivant dans les carrés.]

- Ga: «Donc tu mets 10 là, 10 là et 10 là?»

- Lé: «Voilà, très bien».

- Di : «Donc ça fait 90 ».

- Ga: «20,40, 60, 80, 90. 0k, j'ai compris. Donc alors ...»

$-\mathrm{Di}:$ : $15 \times 6 . »$

- Ga: "Mais il faut s'occuper du prix aussi. »

- Di: "Mais c'est pour cela! 5,95 et il faut combien de narcisses? Il en faut 90. Donc ça fait 6 paquets. Donc c'est 5,95 x 6. » [II prend la calculatrice.] «5,95 x 6 = 35,7.»

- Lé: «Mais oui, mais si ça se met, ça ne marche pas les gars. Parce qu'il nous en faut 20.»

- Di fait semblant de pleurer et dit: «Il faut 20 narcisses par mètre carré et il y a 4,5 $\mathrm{m}^{2}$.»

- Ga: "Oui, mais c'est un paquet de 15. »

- Di : « Oui, et nous il nous en faut 90 narcisses.» 


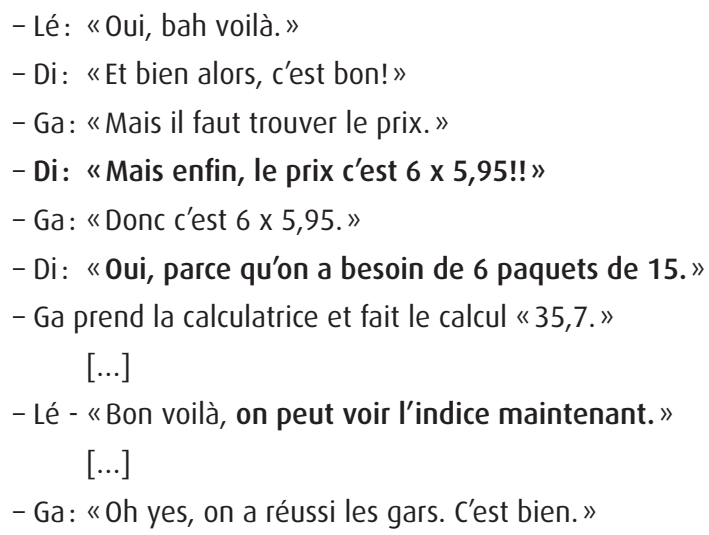

Finalement, les interactions entre élèves, médiatisées par les indices, ont permis de résoudre les quatre nœuds mathématiques et l'analyse des transcriptions montre que trois d'entre eux ont clairement été explicités. La réussite de la tâche de réinvestissement par les trois élèves du groupe témoigne d'un signe d'autorégulation résultant sans doute de la qualité de ces hétérorégulations.

Dans les deux groupes à efficacité modérée (résolution correcte de la tâche en groupe, mais échec d'une partie des membres lors du réinvestissement individuel), l'analyse des interactions montre que les élèves se sont saisis des indices pour dynamiser les échanges, mais qu'ils ont été moins soucieux de s'assurer de la compréhension de chacun des membres.

Dans les deux groupes, la sous-tâche 2 pose quelques difficultés: les élèves estiment que 10 plantes sont nécessaires et se rendent compte de leur erreur à la suite de la lecture de l'indice de vérification. Les deux groupes décident d'utiliser la règle graduée et de tracer une marque à tous les centimètres en vue d'indiquer l'emplacement des plantes. Dans un groupe (G3), la démarche est suivie de près par les trois membres (l'un trace un point tous les centimètres et les deux autres contrôlent le tracé), de sorte que l'on peut considérer que ce nœud mathématique est résolu collectivement. Lors de la tâche de réinvestissement, les trois élèves résolvent correctement cette sous-tâche. Dans l'autre groupe (G2), deux élèves semblent éprouver des difficultés à comprendre pourquoi compter dix fleurs n'est pas correct. L'indice fait office d'argument d'autorité pour rejeter la démarche basée sur le mesurage $(10 \mathrm{~cm}=10$ plantes $)$ puisque la réponse découlant de cette donnée erronée (6,95 euros x 10) s'y trouve (voir transcription 3 - Max constate l'erreur en consultant l'indice de vérification). Un élève (Max toujours) propose alors une démarche correcte, appuyée sur le dessin d'une fleur à chaque centimètre et sur le dénombrement de celles-ci le conduisant à établir qu'il faut en réalité 11 fleurs. Les commentaires d'un élève (Ju) montrent qu'il ne comprend pas (il pense que c'est la latte qui «bugge» (bogue) et revient sur la réponse 10), même s'il finit par accepter la réponse «11» sans écouter l'explication que tente de fournir Max. Le troisième élève (Mat) n'écoute pas non plus et les deux 
élèves (Ju et Mat) finissent par se centrer sur les calculs et sur la réponse finale, sans avoir témoigné de leur compréhension du nœud mathématique. Il n’est dès lors pas surprenant que seul le premier élève (Max) parvienne finalement à résoudre cette partie de la tâche lors de la phase de réinvestissement.

Transcription 3. Extraits des échanges du groupe 2 relatifs à la sous-tâche 2

- Max: «Mince c'est faux!»

- Mat: «Oui, c'est faux alors»

- Ju: «Bah non, c'est juste.» [II prend la latte et le plan et remesure $10 \mathrm{~cm}$.]

- Max: "Mais non, c'est faux si elle met que c'est des réponses fausses. » [...] «Bon attendez, je vais faire ce que je voulais tantôt et on va voir. » [Elle prend une latte et le plan.] «Tu fais comme ça, tu traces une ligne... »

- Mat: «Mais ça fera pareil hein, Max.» [En rigolant.]

- Max: «Mais non, attends. » [Elle trace une ligne.] «Et puis tous les centimètres, tu traces un point.» [Chaque point représentant une fleur.] [...] «Alors y en a combien?» Elle compte les points qu'elle vient de tracer: «1, $2,3 \ldots 10,11 . »$

- Mat: «Ah x 11?»

- Ju: "Bah oui, mais regarde, là, c'est x10 donc c'est la latte qui bugge. » [Il prend la latte et remesure la distance entre les deux points.]

- Mat: "Mais non la latte, elle ne bugge pas.»

- Ju: " C'est x 10, tu vois bien quand même. " [En montrant le 10 de la latte.]

- Max tente d'expliquer: "Mais non, mais on s'en fout des centimètres. Il faut compter. Regarde.» [Elle prend le crayon et avec celui-ci, elle compte les points présents.] «1, 2, 3...»

$[\ldots]$

- Ju n'écoute pas l'explication, puis se saisit de la réponse de Max pour conclure: «0ui, ok, il faut faire x 11. » [II prend alors la calculatrice et dit:] «6,95 x 11.»

- Max termine en même temps son comptage: «... 4, 5, 6, 7, 8, 9, 10, 11. Il y a 11 forsythias. " [Max veut expliquer:] "Parce que j'ai tracé une ligne, et puis fais un trait tous les centimètres. »

- Mat n'écoute pas ce que dit Max et dit fort: «C'est 76,45. Vas-y Max, écrit 76,45.»

Lors de la résolution de la sous-tâche 3, les deux groupes entreprennent au départ une démarche proche de celle mentionnée précédemment. Les deux premiers nœuds sont traités de concert: l'indice de représentation conduit à paver l'aire du triangle à l'aide de carrés ou de demi-carrés, puis les 90 plantes sont comptées par addition de 20 plantes par carré, auxquelles s'ajoutent les 10 plantes nécessaires au demi-carré restant.

Dans un groupe (G3), il y a quelques débats relatifs à la compréhension de la consigne «20 narcisses par $\mathrm{m}^{2}$ » (voir transcription 4), puis deux élèves (La et $\mathrm{Ol}$ ) coopèrent pour mettre en œuvre la procédure décrite ci-dessus. Le troisième (El) semble un peu perdu, comme en témoignent certains de ses commentaires («Mais il faut juste en planter 20 »; «Mais il reste un morceau»). La démarche est verbalisée, mais on peut considérer qu'elle est peu expliquée par les deux élèves qui la dirigent et qu'elle est 
présentée comme une évidence aux yeux du troisième. Lorsque le groupe s'attaque au dernier nœud (le nombre de paquets à acheter), ce sont toujours les deux mêmes élèves qui mènent la danse et se complètent assez efficacement. La demande d'explicitation formulée par le troisième élève (El: «Pourquoi diviser par 15?») n’est pas prise en compte. La consultation de l'indice de vérification renforce la réponse trouvée et les échanges s'arrêtent sans avoir permis d'expliciter clairement la démarche. Aucun élève ne parvient à reproduire cette démarche dans la tâche de réinvestissement face à laquelle tous trois échouent. À la lecture des verbatim, ce résultat n'est pas surprenant pour une élève (El), mais il l'est davantage pour les deux autres ( $\mathrm{La}$ et $\mathrm{Ol})$. Au bout du compte, il semble donc que l'efficacité collective des échanges (coconstruction de la solution par deux élèves) ne se soit pas traduite par une compréhension intra-individuelle, puisque les résultats de la tâche de réinvestissement ne démontrent aucune forme d'autorégulation.

\section{Transcription 4. Extraits des échanges du groupe 3 relatifs à la sous-tâche 3}

- La: "Planter 20 narcisses par $\mathrm{m}^{2}$ dans un parterre de forme triangulaire. Alors il faut regarder... »

- El: «Regardez les filles, I'indice dit qu'il faut 20 narcisses sur $1 \mathrm{~m} 2$.»

- 0l: « 20 narcisses pour $1 \mathrm{~m}^{2}$ donc...»

- El: «Bah il faut 20 narcisses.»

- La: "0ui, mais par mètre carré.»

- Ol: «Donc, tu sais en planter 20, tu sais en planter 40 parce que tu sais mettre un deuxième, $60 . .$. » [En montrant avec son doigt sur l'indice de représentation.]

- El: "Mais il faut juste en planter 20. Regardez l'énoncé: planter 20 narcisses. »

- La: "Par mètre carré, donc par mètre carré. » [En effectuant un geste de la main pour dire qu'il y a aura plusieurs mètres carrés.]

- Ol: «Oui par mètre carré. Donc 20,40,60, ça plus ça, ça fait 80 et il reste un demi donc ça fait 10.»

- El: «Mais il reste un morceau.»

- La: "C'est 10 narcisses car c'est la moitié.»

- Ol: "Donc ça fait 90 narcisses.»

- La: "Oui, donc les narcisses, c'est 5,95 le paquet de 15. Et pour arriver à 90, on fait ...»

- 0l: «Euh bah ça ne marche pas. C'est quoi le calcul?»

- La: « 90 divisé en 15.»

- Ol: Écrit le calcul sur la feuille et ensuite, le fait à la calculatrice: "Ah mais si, ça marche. Ça fait 6.»

- El: "Pourquoi divisé en 15?»

- La (qui n'a pas prêté attention à la question d'El): "Donc si ça fait 6 . Il faut faire 6 x 5,95. »

- 0l: " Oui, tu as raison. » [Elle écrit le calcul sur la feuille ( 6 x 5,95»), fait le calcul à la calculatrice et dit:] «Ça fait 35,7.»

- El retourne l'indice de résolution.

- ol se penche pour regarder l'indice et dit: "Je pense que l'on a juste. Maintenant, on doit ... »

Dans l'autre groupe (G2), l'un des membres (Ju) témoigne de son incompréhension du $2^{\mathrm{e}}$ nœud mathématique ( Pourquoi 90 alors qu'il en faut 20?»), mais son questionnement n'est pas pris en compte par les deux autres. Un autre élève (Max) exprime la 
confusion que lui procure le $3^{\mathrm{e}}$ nœud («Mais il y en a 15 dans un paquet!») et le troisième (Mat) s'en saisit pour proposer une stratégie de comptage par 15 pour arriver à 90 . Le nombre «6" est ainsi trouvé et le problème résolu, mais un élève a dirigé le raisonnement, un autre a effectué les calculs et le troisième a noté les solutions. Dans ce groupe, la distribution des rôles n'a pas permis de réels échanges constructifs: les hétérorégulations étaient peu nombreuses et peu ciblées sur les nœuds mathématiques. En fin de compte, un seul élève a pu résoudre cette partie de la tâche complexe lors de la phase de réinvestissement.

Si l'on s'intéresse maintenant au groupe qui n'est pas parvenu à résoudre entièrement la tâche complexe, on notera que les élèves se sont fourvoyés dans la résolution de la sous-tâche 3 : ils n'ont pas compris l'indice de représentation «20 narcisses par $\mathrm{m}^{2}$ » et l'ont interprété comme ne nécessitant pas de calcul («L'indice dit que par $\mathrm{m}^{2}$, il en faut 20, et donc on n'a pas besoin de calculer.») car il signifiait pour eux qu'il faut «20 fleurs en tout». Les débats ont ensuite porté sur la façon de calculer le prix de 20 fleurs lorsqu'on connaît celui de 15 fleurs, mais les élèves n'ont jamais pris en compte le fait qu'elles étaient vendues par 15. Ils ont effectué une division pour calculer le prix de 5 fleurs et ont ajouté ce prix (1,98 euro) au prix d'un bac de 15 (5,95 euros). Cette erreur ne figurant pas parmi les exemples de solutions erronées, leur processus de vérification n'a pas été poussé plus loin. Assez logiquement, ils échouent tous les trois à cette partie de la tâche complexe lors de la phase de réinvestissement.

\section{DISCUSSION}

Concernant la première question de recherche, "Comment les interactions entre élèves, médiatisées par les indices, permettent-elles aux élèves de résoudre les "nœuds mathématiques" impliqués dans la tâche complexe?», les résultats montrent un impact positif des interactions entre élèves puisqu'au bout du compte 3 groupes sur 4 réussissent l'ensemble de la tâche complexe, le quatrième groupe échouant uniquement à l'une des sous-tâches. L'analyse des transcriptions montre que les indices influencent les hétérorégulations: ce sont eux qui aident à percevoir ou à surmonter la plupart des nœuds mathématiques. Globalement, l'impact des indices s'avère comparable d'un groupe à l'autre, mais variable d'une sous-tâche à l'autre.

Dans la sous-tâche 2, le nœud mathématique se situe au niveau du problème d'intervalle consistant à comptabiliser 11 plantes (et non 10). L'indice de représentation ne suffit pas à permettre à tous les élèves de conscientiser l'idée selon laquelle devoir compter les deux plantes mises aux extrémités nécessite d'ajouter 1 à la mesure de la distance entre ces deux extrémités. Ainsi, même si un élève du groupe a trouvé la solution correcte, ce n'est généralement pas suffisant pour en convaincre les autres. Dans ce cas, le conflit sociocognitif (Buchs et al., 2008) lié à la constatation de réponses différentes ne fonctionne pas. C'est l'indice de vérification qui déstabilise le 
groupe et provoque le conflit cognitif nécessaire à la recherche d'une autre démarche et à la résolution du nœud mathématique.

Dans la sous-tâche 3, l'indice de représentation dirige tous les groupes vers le développement d'une stratégie de pavage de la surface en carrés et en demi-carrés conduisant à comptabiliser 20 plantes par carré, sans jamais utiliser de formule pour calculer l'aire de la surface. En procédant de cette façon, les deux premiers nœuds mathématiques (calcul de l'aire du triangle et calcul du nombre de plantes nécessaires) sont traités de concert dans une démarche qui s'avère généralement efficace. Une fois les 90 plantes trouvées, le troisième nœud consistant à calculer qu'il convient d'acheter 6 paquets de 15 plantes semble généralement poser peu de difficultés aux groupes, et ceci même si l'on peut regretter que la façon de calculer le «6» n'est pas toujours suffisamment explicitée et réexpliquée pour s'assurer de la compréhension de chacun des élèves. Dans cette sous-tâche, les indices de vérification n'ont pas joué de rôle majeur puisque trois groupes sur quatre ont trouvé directement la solution correcte, généralement en s'appuyant sur la collaboration efficace de deux élèves, et que le dernier groupe a produit une erreur ne figurant pas parmi les indices relevés. C'est d'ailleurs là que se situe la limite principale des indices de vérification proposés: ils ne fonctionnent évidemment que si les groupes produisent l'erreur ciblée. Dans le cas contraire, ils jouent même un rôle contre-productif, puisque la constatation de la non-correspondance entre la réponse trouvée et l'indice joue un rôle de validation auprès du groupe, qui ne cherche dès lors nullement à mettre en œuvre un autre type de procédure de vérification. Même si les indices avaient été construits sur la base des réponses erronées les plus fréquemment rencontrées dans une étude préalable (Demonty et al., 2014), ils ne rendent évidemment pas compte de toutes les erreurs possibles. Le choix avait été fait de ne proposer que deux erreurs pour ne pas surcharger cognitivement les élèves. Un enjeu des indices de vérification est d'enclencher un processus autorégulateur en aidant les élèves à prendre conscience des contraintes du problème. En ce sens, la démarche erronée produite par le dernier groupe est intéressante dans la mesure où elle court-circuite complètement les trois nœuds mathématiques (pas de calcul de l'aire du triangle, pas de calcul du nombre total de narcisses et pas de prise en compte de la vente par paquets) pour procéder à une simple proportionnalité permettant de calculer le prix de 20 fleurs au départ du prix connu pour 15 d'entre elles.

Concernant la deuxième question de recherche («Les élèves qui ont pu résoudre correctement la tâche complexe en groupe parviennent-ils à réinvestir ces acquis collectifs dans un travail individuel du même type?»), force est de constater que les résultats sont nettement moins encourageants puisque ce n'est que dans un seul groupe que les interactions entre élèves semblent avoir réellement joué un rôle d'hétérorégulations favorisant l'autorégulation. L'analyse des transcriptions montre que le groupe pour lequel la phase de réinvestissement a été réussie par tous les élèves est le seul dans lequel les élèves ont pris le temps de discuter précisément des différents nœuds mathématiques (même si le dernier est resté un peu plus implicite) et 
de s'assurer, comme les consignes le demandaient, de veiller à la compréhension de chacun et à la promotion d'interaction de qualité (Gillies, 2016; Plante, 2012). C'est d'ailleurs sans doute là que se situe la lacune principale de l'approche développée: on peut en effet penser que la responsabilité individuelle n'était pas suffisamment contrainte pour assurer un fonctionnement optimal du groupe et assurer les apprentissages de chacun des élèves.

\section{CONCLUSION}

Malgré le caractère exploratoire de l'étude et le faible nombre de groupes concernés par l'analyse, il nous semble que l'approche proposée - un dispositif d'apprentissage coopératif structurant les interactions entre élèves grâce à des indices conçus en fonction des enjeux mathématiques des tâches proposées - offre un potentiel intéressant pour soutenir le développement des compétences des élèves en résolution de problèmes. Ce type d'approche pédagogique, qui permet aux élèves de rester acteurs de leurs apprentissages en réalisant un travail autonome, soutenu par des «outils» favorisant les hétérorégulations comme sources potentielles d'autorégulation, semble constituer une voie intéressante dont pourraient assez aisément se saisir les enseignants et les enseignantes. À nos yeux, en toute cohérence avec le présent numéro thématique, l'enjeu principal de l'article était non seulement de donner à voir la potentielle richesse des interactions entre élèves ainsi structurées, mais aussi de pointer les difficultés et les limites liées au travail de groupe, et ceci même quand celui-ci a été conçu en s'appuyant sur une approche méthodologique (l'apprentissage coopératif) dont l'efficacité a été largement démontrée (Buchs et al., 2012; Gillies, 2016; Plante, 2012; Slavin, 2015). Par ailleurs, l'efficacité du dispositif coopératif envisagé devrait a priori pouvoir s'accroître si les enseignants et les enseignantes le soutenaient en y apportant de potentielles hétérorégulations (Allal, 2007). Toutefois, encore faut-il que ces enseignants ou enseignantes trouvent comment interagir dans «les processus de pensée et de communication en cours» (Perrenoud, 1998, p. 114) et se sentent autorisés à mettre en œuvre un «étayage» (Mottier Lopez, 2012) qui ne prend pas pour autant la forme d'un cadrage «trop étroit» (Bonnery, 2009).

\section{Références bibliographiques}

ABRAMI, Ph., CHAMBERS, B., POUlSEN, C., De SIMONE, C., D'APOLlONIA, S. et HOWDEN, J. (1996). Apprentissage coopératif. Théories, méthodes, activités. Québec: Éditions de la Chennelière. 
ALLAL, L. (2007). Régulations des apprentissages: orientations conceptuelles pour la recherche et la pratique en éducation. Dans L. Allal et L. Mottier Lopez (dir.), Régulation des apprentissages en situation scolaire et en formation (p. 7-23). Bruxelles: De Boeck.

BAUDRIT, A. (2007). Apprentissage coopératif/Apprentissage collaboratif: d'un comparatisme conventionnel à un comparatisme critique. Les Sciences de l'éducation - Pour l'Ère nouvelle, 40(1), 115-136.

BONNERY, S. (2009). Scénarisation des dispositifs pédagogiques et inégalités d'apprentissage. Revue française de pédagogie, 167, 13-23.

BUCHS, C., DARNON, C., QUIAMZADE, A. MUGNY, G. et BUTERA, F. (2008). Conflits et apprentissage. Régulation des conflits sociocognitifs et apprentissage. Revue française de pédagogie, 163, 105-125.

BUCHS, C., LEHRAUS, K. et CRAHAY, M. (2012). Coopération et apprentissage. Dans M. Crahay (dir.), L'école peut-elle être juste et efficace? (p. 421-454). Bruxelles: De Boeck.

CHEN, C.H. et CHIU, C.H. (2016). Collaboration scripts for enhancing metacognitive self-regulation and mathematics literacy. International Journal of Science and Mathematics Education, 14, 263-280.

CRAHAY, M., HINDRYCKX, G. et LEBE, M. (2001). Analyse des interactions entre enfants en situation de tutorat portant sur des problèmes mathématiques de type multiplicatif. Revue française de pédagogie, 136, 133-145.

DEMONTY, I. et FAGNANT, A. (2014). Tâches complexes en mathématiques: difficultés des élèves et exploitations collectives en classe. Éducation et Francophonie, XLII(2), 173-189.

FAGNANT, A., DUPONT, V. et DEMONTY, I. (2016). Régulation interactive et résolution de tâches complexes en mathématiques. Dans L. Mottiez Lopez et W. Tessaro (dir.), Les processus de jugement dans des pratiques d'évaluation des apprentissages (p. 229-551). Berne: Peter Lang.

FAGNANT, A., MARCOUX, G. et VLASSIS, J. (2014). Résolution de problèmes mathématiques et développement de compétences: comment soutenir les élèves dans leur apprentissage? Cahiers des Sciences de l'Éducation, 36, 1-6.

GILLIES, R.M. (2016). Cooperative learning: Review of research and practice. Australian Journal of Teacher Education, 41(3), 39-54. 
GINSBURG, A., COOKE, G., LEINWAND, S. et POLLOCK, E. (2005). Reassessing U.S. international mathematics performance: New findings from 2003 TIMSS and PISA. Washington, D.C.: American institute for Research.

HANIN, V. etVAN NIEUWENHOVEN, C. (2016). Évaluation d'un dispositif pédagogique visant le développement de stratégies cognitives et métacognitives en résolution de problèmes en première secondaire. Évaluer.Journal international de Recherche en Éducation et Formation (e-jiref), 2, 53-88.

HATTIE, J. (2009). Visible learning: A synthesis of over 800 meta-analyses relating to achievement. London: Routledge.

JAEGERS, D., LAFONTAINE, D. et FAGNANT, A. (2016). Favoriser la co-régulation et la co-construction d'une démarche efficace de résolution de problèmes mathématiques en fin d'enseignement primaire. Revue Suisse des Sciences de l'Éducation, 38(3),569-589.

LERHAUS, K. (2002). La pédagogie coopérative: de la formation à la mise en pratique. Revue Suisse des Sciences de l'Éducation, 24(3), 1-22.

LEMAIRE, E. (2016). Face à une tâche complexe, les modalités de travail données aux différents groupes influencent-elles les interactions sociocognitives et l'efficacité des groupes (Mémoire de Master inédit). Université de Liège.

MERCIER-BRUNEL, Y. (2017). Soutenir l'autorégulation des apprentissages en séance de correction collective. Dans S. Cartier et L. Mottier Lopez (dir.), Soutien à l'apprentissage autorégulé en contexte scolaire: perspectives francophones (p. 137159). Québec: Presses de l'Université du Québec.

MEVARECH, Z.R. et KRAMARSKI, B. (2014). Critical Maths for Innovative Societies: The Role of Metacognitive Pedagogies. Paris: OECD publishing.

MOTTIER LOPEZ, L. (2012). La régulation des apprentissages en classe. Bruxelles: De Boeck.

MOTTIER LOPEZ, L. (2015). Évaluation-régulation interactive: étude des structures de participation guidée entre enseignant et élèves dans le problème mathématique «Enclos de la chèvre». Mesure et évaluation en éducation, 381, 89-120.

PLANTE, I. (2012). L’apprentissage coopératif: des effets positifs sur les élèves aux difficultés liées à son implantation en classe. Revue Canadienne de l'Éducation, 35(3), 252-283.

PERRENOUD, P. (1998a). L'évaluation des élèves. De la fabrication de l'excellence à la régulation des apprentissages: entre deux logiques. Bruxelles: De Boeck. 
SANDRON, L. et FAGNANT, A. (2017). L'influence d'une formation au tutorat sur les performances en résolution de problèmes et sur la motivation autodéterminée d'élèves de fin d'enseignement primaire. Revue Française de Pédagogie, 198, 75-92.

SLAVIN, R.E. (2015). Cooperative learning in elementary schools. Education 3-13, 43(1), 5-14.

SLAVIN, R. E. et LAKE, C. (2008). Effective programs in elementary mathematics: A best-evidence synthesis. Review of Educational Research, 78(3), 427-515.

THEVENOT, C., BARROUILLET, P. et FAYOL, M. (2015). De l'émergence du savoir calculer à la résolution des problèmes arithmétiques verbaux. Dans M. Crahay et M. Dutrevis (dir.), Psychologie des apprentissages scolaires (p. 169-197). Bruxelles: De Boeck.

VAN DOOREN, W., VERSCHAFFEL, L., GREER, B., De BOCK, D. et CRAHAY, M. (2015). La modélisation et la résolution de problèmes d'application. Dans M. Crahay et M. Dutrevis (dir.), Psychologie des apprentissages scolaires (p. 199-220). Bruxelles: De Boeck. 


\section{Annexe 1.}

\section{TÂCHE COMPLEXE, "NGUDS MATHÉMATIQUES " ET INDICES DE REPRÉSENTATION ET DE RÉSOLUTION}

Note: La tâche complexe proposée est très largement inspirée de la tâche éponyme proposée par la commission d'outils d'évaluation et disponible sur le site du ministère de I'Enseignement (http://www. enseignement.be). Sur le plan donné aux élèves, les éléments à mesurer conduisaient à des nombres entiers (ou à des décimaux limités aux dizièmes).

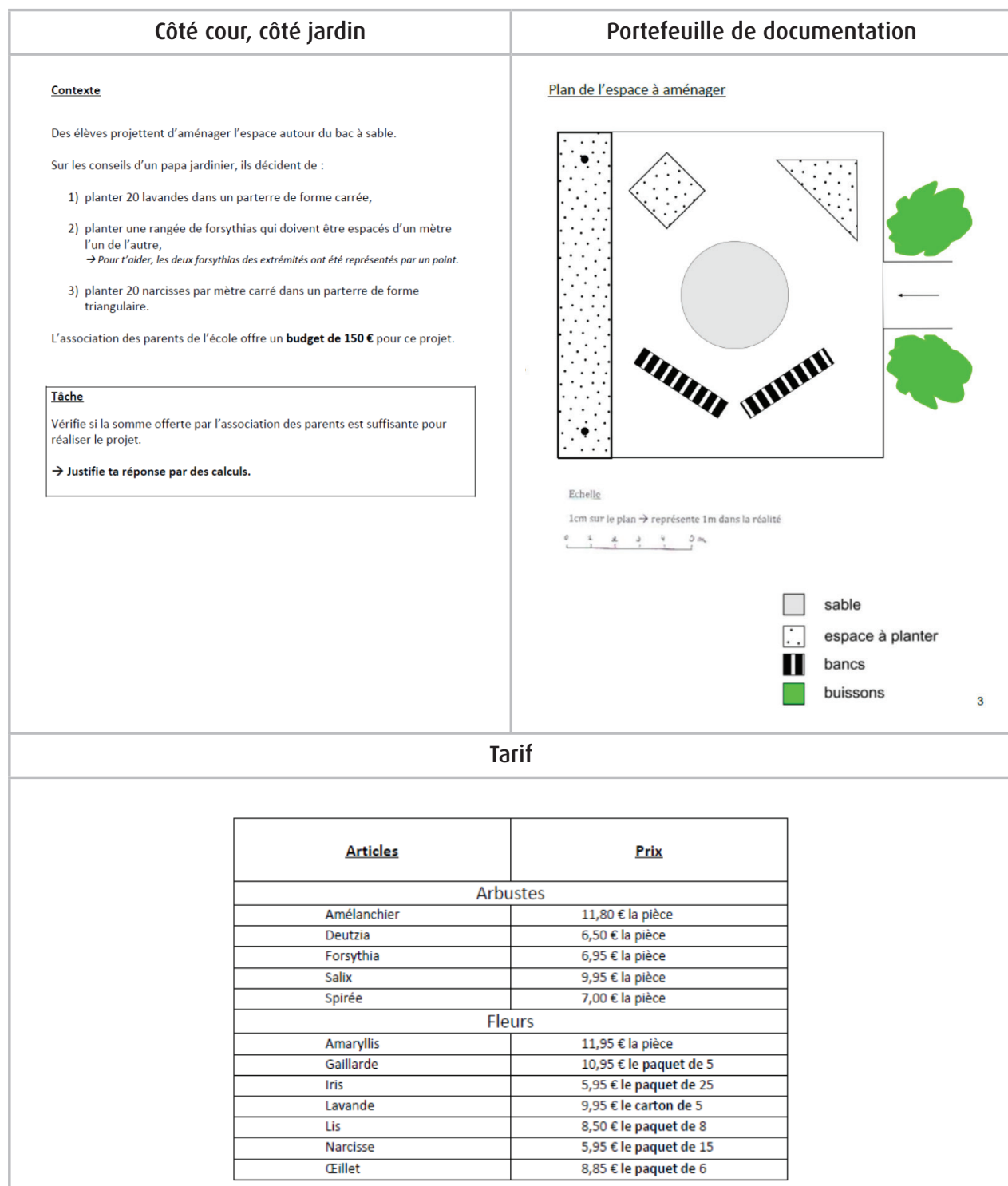

Les 4 "nœuds mathématiques » principaux, tels que révélés par une étude préalable (Demonty et al., 2014):

Sous-tâche 2 - $\boldsymbol{1}^{\text {er }}$ nœud - Prendre en compte qu'il s'agit d'une situation d'intervalle: pour placer une plante tous les $\mathrm{m}$ (ou $\mathrm{cm}$ ), il convient d'ajouter « +1 » à la valeur de la mesure entre les deux extrémités (10 cm sur le plan donné aux élèves en taille réelle).

Sous-tâche 3 - $\boldsymbol{1}^{\text {er }}$ nœud - Comprendre qu'il faut s'intéresser à l'aire du triangle et la calculer $\left(4,5 \mathrm{~m}^{2}\right)$;

$2^{e}$ nœud - Calculer le nombre total de fleurs $\left(4,5 \mathrm{~m}^{2} \times 20\right.$ fleurs $/ \mathrm{m}^{2}=90$ fleurs $)$;

$3^{e}$ nœud - Calculer le nombre de paquets à acheter (15 fleurs/paquet $\mathrm{x} 6$ paquets $=90$ fleurs) 


\section{Les indices de représentation et de vérification pour la sous-tâche 2}

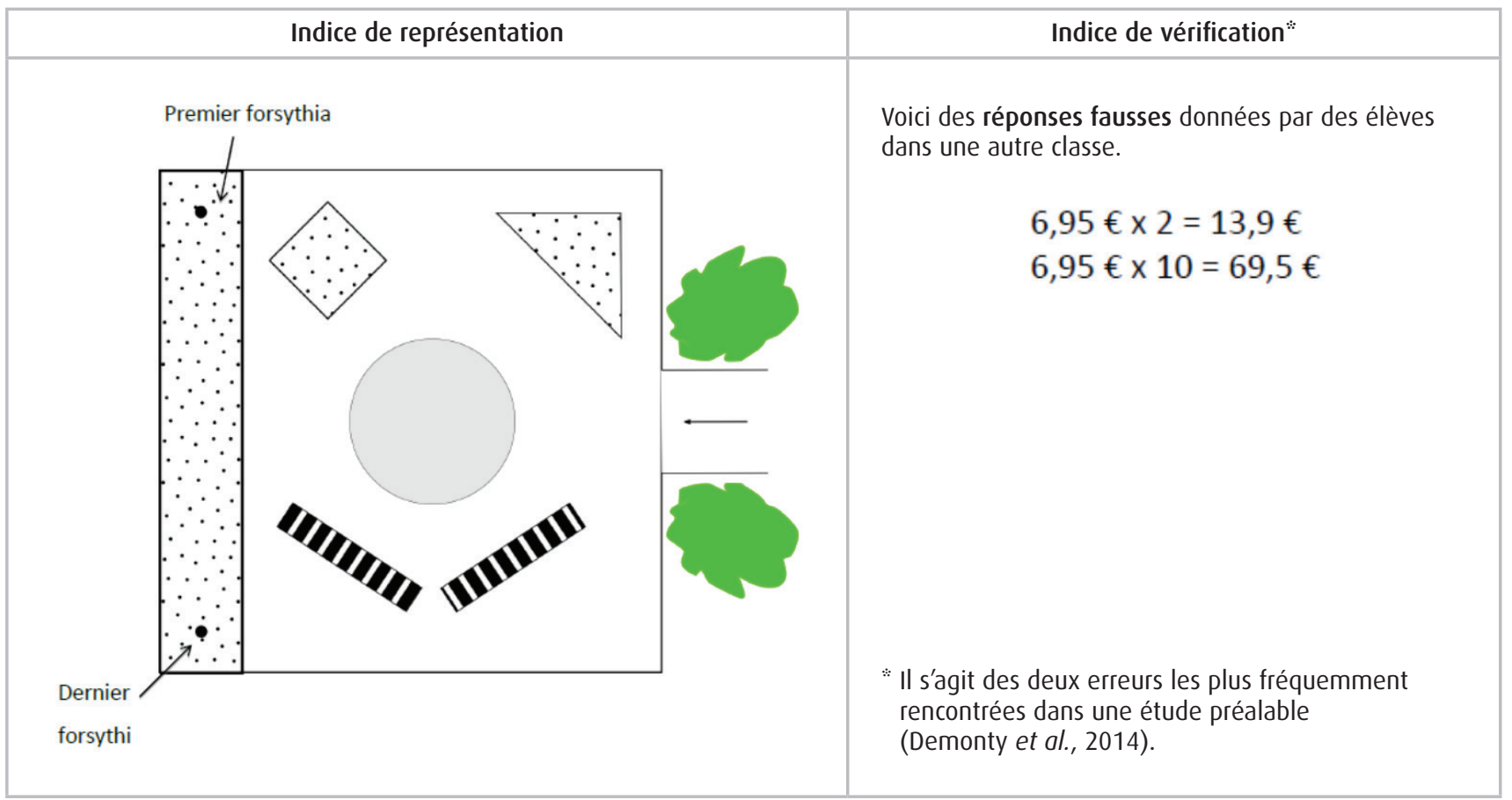

Les indices de représentation et de vérification pour la sous-tâche 3

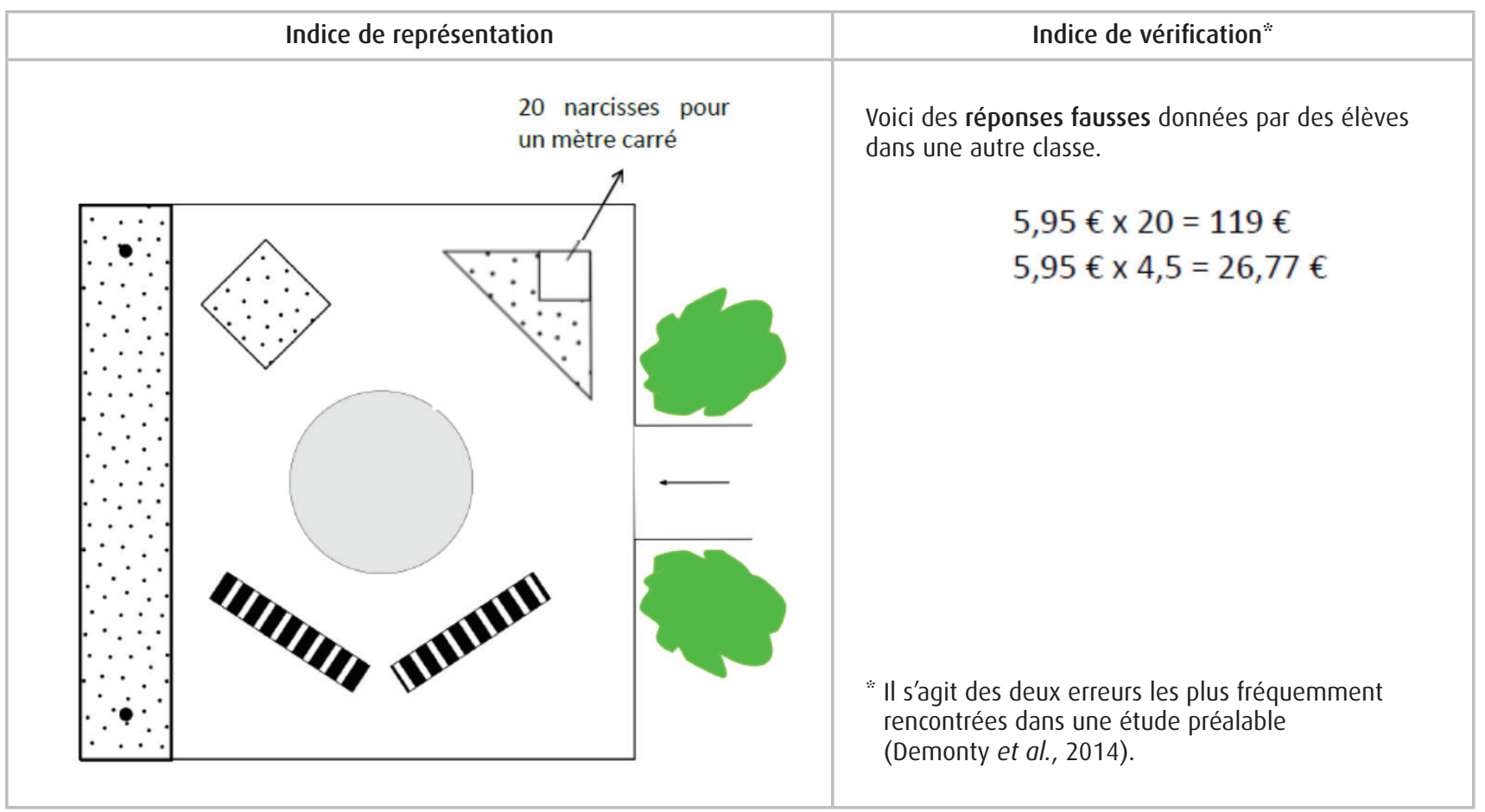




\section{Annexe 2.}

\section{SCRIPT LU AUX ÉLÈVES AU DÉBUT DU TRAVAIL DE GROUPE COOPÉRATIF}

"Je vous ai placés par groupes de trois élèves. Je vais vous distribuer le même problème que la fois passée car il s'agit d'une tâche complexe et j'aimerais voir si le fait de travailler ensemble va permettre une meilleure réussite que lorsque vous avez travaillé seul. Le groupe est constitué de cette façon car vous n'aviez pas les mêmes réponses donc j'aimerais que vous en discutiez et que vous réalisiez le problème ensemble. Tout d'abord, l'un de vous va lire le problème en entier au groupe pour vous le remettre en mémoire. Ensuite, je vais vous demander pour chaque sous-tâche marquée par un numéro de respecter un rôle bien précis. J'espère que cela pourra vous aider. Je vais vous expliquer les différents rôles.

- Rôle 1: Un enfant lit la sous-tâche et réexplique ce que vous devrez faire. Ensuite, il cherchera l'info dans les documents. Où faut-il regarder pour répondre à cette question? De quoi avez-vous besoin? ...

- Rôle 2: Un autre élève sera le responsable des indices et sera chargé de les gérer et de les utiliser au bon moment. En effet, les indices vont vous aider à comprendre le problème. L'indice 1 est celui de représentation, il peut être utilisé directement après la lecture de la sous-tâche. Par contre, l'indice 2, celui de résolution, vous ne pourrez l'utiliser qu'une fois que vous avez trouvé une réponse à la sous-tâche. Une fois que l'élève qui a le rôle 1 a lu l'énoncé et cherché les éléments d'informations importantes pour résoudre la sous-tâche, vous discuterez ensemble quant à la (aux) démarche(s) à effectuer pour résoudre le problème et vous écrirez tous vos calculs sur la feuille prévue à cet effet. Une fois que vous avez trouvé une réponse, l'indice 2 peut être regardé.

- Rôle 3: Le troisième enfant aura le rôle de "vérificateur» c'est-à-dire qu'il s'assurera que chacun a compris le procédé pour résoudre la sous-tâche et que tout le monde est d'accord avec le choix de réponse. Il s'occupera également de vérifier les calculs et les réponses.

Chacun doit être responsable de son rôle. Je vous rappelle également qu'il s'agit d'un travail coopératif donc si l'un de vous n'arrive pas à mener à bien le rôle défini, les autres, vous pouvez bien sûr l'aider. Pour la première sous-tache, $\mathrm{X}$ jouera le rôle de lire et de rechercher les informations utiles; $\mathrm{Y}$ jouera le rôle du responsable des indices et $\mathrm{Z}$ jouera le rôle de vérificateur. À chaque sous-tâche à réaliser vous changerez de rôle. Vous allez démarrer avec la sous-tâche 1. Quand vous aurez terminé cette première sous-tâche, on changera les rôles de chacun. Je vous expliquerai à ce moment-là. Comme la fois dernière la calculatrice est autorisée, à vous de l'utiliser à bon escient. Avez-vous encore des questions? 
Une dernière chose, je vais vous filmer pour garder une trace de vos échanges, mais faites comme si la caméra n'était pas là. Ce n'est pas un contrôle donc soyez détendus. Je vous rappelle qu'il s'agit d'un travail de groupe donc que si vous avez des questions, vous les posez à vos camarades et non à moi. De plus, lors d'un travail en coopération certaines valeurs sont à appliquer comme le respect, l'entraide, la solidarité, le droit à l'erreur ... Bon travail.» (Extrait de Lemaire, 2016, Annexes, p. 6-7.). 